Results $5 \alpha \mathrm{R} 1$ expression in human livers was parenchymal and greater in non-alcoholic steatohepatitis (NASH) livers compared to normal liver and was associated with greater lobular inflammation. $5 \alpha$ R1 gene expression was not affected by NASH severity. ALIOS induced steatohepatitis $(6 \mathrm{~m})$ and significant fibrosis (F3 at $12 \mathrm{~m}$ ) in WT and $5 \alpha \mathrm{R} 1 \mathrm{KO}$ mice. Compared with WT mice, $5 \alpha \mathrm{R} 1 \mathrm{KO}$ mice fed the ALIOS diet demonstrated significantly greater liver weight, steatosis score (median 3 vs 1), and hepatic triglyceride accumulation by 6 months (93.1 vs $62.4 \mathrm{nmol} / \mathrm{mg} \mathrm{p}=0.002$ ). mRNA expression of CPT1 $\alpha$, a key enzyme in hepatic fatty acid $\beta$ oxidation, was reduced in $5 \alpha \mathrm{R} 1^{-/-}$mice. There was a trend $(p=0.1)$ towards worse inflammation (Kleiner lobular inflammation/qPCR for hepatic TNFa) at 6 months in $5 \alpha \mathrm{R} 1 \mathrm{KO}$ mice, but no difference in inflammation or fibrosis at 12 months despite the presence of greater hepatic steatosis. The number of panCK-positive cells observed in WT mice fed ALIOS diet increased significantly with longer duration (0 $\mathrm{m} \mathrm{0.15 \% ,} 6 \mathrm{~m} \mathrm{0.44 \% ,} 12 \mathrm{~m} \mathrm{0.94 \% ,} \mathrm{p=0.028)} \mathrm{and} \mathrm{at}$ 12 months was significantly greater than mice fed normal chow (0.94\% vs $0.18 \% \mathrm{p}=0.03$ ). Despite the absence of cirrhosis $5 / 10 \mathrm{WT}$ mice developed dysplasia/hepatocellular carcinoma after ALIOS for 12 months vs $0 / 55 \alpha \mathrm{R} 1 \mathrm{KO}$ mice. $5 \mathrm{aR} 1 \mathrm{KO}$ mice had lower numbers of panCK-positive cells ( $0.62 \%$ vs $0.94 \%$ ) compared to its WT.

Conclusion ALIOS diet induces the entire spectrum of NAFLD from simple steatosis to advanced NASH with fibrosis and HCC, with a mounting oval cell response to increasing duration of diet. $5 \alpha \mathrm{R} 1 \mathrm{KO}$ promotes hepatic steatosis in the absence of worsening fibrosis, attenuates the oval cell response and exerts a protective effect on hepatocarcinogenesis thereby demonstrating the role of $5 \alpha \mathrm{R}$ in NAFLD pathogenesis.

\section{P87 VASCULAR ADHESION PROTEIN-1 PROMOTES INCREASES IN LIVER INFILTRATING CD4+ T CELLS AND NKT CELLS AND INDUCTION OF FIBROGENESIS IN STEATOHEPATITIS}

doi:10.1136/gutjnl-2011-300857a.87

${ }^{1} \mathrm{~L}$ C Claridge, ${ }^{1} \mathrm{C}$ J Weston, ${ }^{1} \mathrm{E}$ L Haughton, ${ }^{2} \mathrm{~N}$ Westerlund, ${ }^{1} \mathrm{G}$ M Reynolds, ${ }^{1} \mathrm{P} F$ Lalor, ${ }^{3} \mathrm{~J}$ W Tomlinson, ${ }^{2} \mathrm{D} J$ Smith, ${ }^{4} \mathrm{C}$ P Day, ${ }^{1} \mathrm{D}$ H Adams. ${ }^{1} \mathrm{NIHR}$ Biomedical Research Unit and Centre for Liver Research, University of Birmingham, UK; ${ }^{2}$ Biotie Therapies Corp., Turku, Finland; ${ }^{3}$ Centre for Obesity Research, University of Birmingham, UK; ${ }^{4}$ Institute of Cellular Medicine, Newcastle University, Newcastle, UK

Introduction Vascular Adhesion Protein-1 (VAP-1) is an adhesion molecule and membrane-bound amine oxidase. Our group has previously demonstrated that VAP-1 is able to support lymphocyte recruitment across human hepatic sinusoidal endothelium in vitro. Soluble VAP-1 (sVAP-1) is released into the circulation from adipose tissue and the hepatic vascular bed, has insulin like effects and is capable of inducing and propagating oxidative stress.

Aim To investigate the role of VAP-1 in the progression of steatosis to steatohepatitis and cirrhosis.

Method Human hepatic VAP-1 expression was determined using immunofluorescent labelling and multicolour confocal microscopy. Serum sVAP-1 levels were measured by ELISA in 144 patients with histologically graded NAFLD and 74 controls matched for age and metabolic phenotype. Two murine models of steatohepatitis were studied; (1) a methionine choline deficient diet was administered for 6 weeks in wild-type (WT) mice, WT mice treated with anti-VAP-1 antibody, and VAP-1 null mice ( $\mathrm{n}=6$ per group), 2) A western lifestyle model incorporating high trans-fat diet and fructose supplemented drinking water was administered to WT and VAP-1 null mice for 6,9 and 12 months ( $n=10$ per group). Control animals remained on normal chow. Liver infiltrating lymphocytes were identified and quantified using flow cytometry. Fibrosis was assessed by immunohistochemistry and qRT-PCR for aSMA and collagen I. Results (1) Human studies: We report increased hepatic expression of VAP-1 in NAFLD associated with elevated serum levels of sVAP-1 compared with controls $(946 \pm 468 \mathrm{ng} / \mathrm{ml}$ vs $265 \pm 78 \mathrm{ng} / \mathrm{ml}$, $\mathrm{p}<0.0001)$. Multiple regression modelling reveals sVAP-1 to be the best predictor of histological fibrosis in our cohort. We detected strong VAP-1 expression in fibrotic septa co-localised with activated liver myofibroblasts (aLMF). In vitro human aLMF expressed and released sVAP-1 which promoted lymphocyte migration through a novel $\mathrm{H}_{2} \mathrm{O}_{2}$-mediated enzyme-dependent mechanism. (2) Experimental steatohepatitis: VAP-1 null and/or antibody treated mice developed fewer inflammatory foci and delayed onset of fibrosis in two murine models of NASH. In both models there was a specific failure to expand the intrahepatic CD4+ and NKT cell populations in VAP-1 null mice compared with 2 to 3-fold increases in WT mice. Three WT animals developed hepatocellular carcinoma by 12 months in the western lifestyle model but no tumours were found in VAP-1 null mice.

Conclusion Our results implicate an important role for VAP-1 in steatohepatitis, in both humans and mice. The ability to target VAP1 with antibodies or small molecule inhibitors makes it an attractive therapeutic target.

\section{P88 VASCULAR ADHESION PROTEIN-1 (VAP-1) MODULATES GLUCOSE AND LIPID UPTAKE IN NON-ALCOHOLIC FATTY LIVER DISEASE (NAFLD)}

doi:10.1136/gutjnl-2011-300857a.88

${ }^{1} \mathrm{~S}$ Karim, E Liaskou, ${ }^{1} \mathrm{~J}$ Youster, ${ }^{2} \mathrm{Fei}-\mathrm{L}$ Lim, ${ }^{2} \mathrm{~K}$ MacAulay, ${ }^{3} \mathrm{~S}$ Jalkanen, ${ }^{1} \mathrm{D} \mathrm{H}$ Adams, ${ }^{1} \mathrm{P} F$ Lalor. ${ }^{1}$ University of Birmingham; ${ }^{2}$ Unilever Discover; ${ }^{3}$ University of Turku

Introduction NAFLD is characterised by steatosis, chronic inflammation and fibrosis. The underlying mechanisms include insulin resistance, increased free fatty acid flux from de-novo lipogenesis and decreased lipid oxidation. Vascular Adhesion Protein-1 (VAP -1 ), is an adhesion molecule with semicarbazide- sensitive amine oxidase activity (SSAO), which is also expressed as a soluble protein in serum (sVAP-1) and elevated in inflammatory liver diseases such as NAFLD. VAP-1 has been shown to modulate glucose and lipid uptake in muscle and adipose tissue and thus we investigated whether it may contribute to glucose and lipid homeostasis in human liver tissue.

Method We have used precision cut liver slices (PCLS) from normal and diseased human liver specimens and cultured human sinusoidal endothelium (HSEC) and hepatocyte cells in combination with VAP-1 substrates (200 $\mu \mathrm{M}$ methylamine or benzylamine) and inhibitors ( $400 \mu \mathrm{M}$ bromoethylamine) to perform standard ex vivo radiolabelled glucose uptake and fatty acid uptake assays using oil red $O$ quantification following exposure of cells to Oleic and Palmitic acid (PA). Immunohistochemical staining and qPCR were performed using standard techniques and for confirmatory experiments HSEC were transfected with enzymatically active/inactive VAP1.

Results OPCR confirmed upregulation of VAP-1 mRNA ( $\Delta \Delta \mathrm{CT}$ $=1.144 \mathrm{p}=0.03)$ in NASH vs normal liver and also changes in FABP1, $-4,-5$, FATP3, -4 ( $\mathrm{p} \leq 0.05$ for all) and GLUT-1, 2, 3, 5, 8, 9 and 12 in NAFLD compared to normal individuals. Results were confirmed using immunohistochemical staining. Exposure of human PCLS to sVAP- 1 and methylamine typically resulted in a $38-54 \%$ increase in PA uptake ( $\mathrm{p} \leq 0.01$ for all) and a $20 \%$ increase in hepatocyte glucose uptake in vitro which could be inhibited using bromoethylamine. Transduction of HSEC with enzymatically active VAP-1 also increased glucose uptake which was prevented in the absence of enzyme activity. Interestingly methylamine treatment of human liver resulted in decreased expression of mRNA for glucose transporters and an increase in some lipid transporters including FABP6, FATP and LRP8, and $\mathrm{H}_{2} \mathrm{O}_{2}$ produced by SSAO activity increase lipid uptake by hepatic cells. 
Conclusion In conclusion, we demonstrate for the first time global alterations in cellular expression of glucose and lipid transporter proteins in human NAFLD. We confirm that VAP-1 is elevated in disease and that SSAO activity of VAP-1 results in enhanced hepatic lipid and glucose uptake and changes in transporter expression. Thus we propose that bioactive metabolites of SSAO activity contribute to the metabolic derangement evident in fatty liver disease.

\section{\begin{tabular}{|l|l}
\hline P89 OSTEOPONTIN PROMOTES LYMPHOCYTE RECRUITMENT IN \\
\hline
\end{tabular} STEATOHEPATITIS}

doi:10.1136/gutjnl-2011-300857a.89

${ }^{1} \mathrm{E}$ Liaskou, 'L C Claridge, ${ }^{1} \mathrm{H}$ Shah, ${ }^{1} \mathrm{Y}$ Oo, ${ }^{1} \mathrm{~J}$ Shaw, ${ }^{2} \mathrm{Z} \mathrm{Mi},{ }^{2} \mathrm{P}$ C Kuo, ${ }^{3} \mathrm{~A}$ Canbay, ${ }^{4} \mathrm{~A}$ M Diehl, ${ }^{1} \mathrm{D}$ H Adams, ${ }^{1}$ Wing-Kin Syn. ${ }^{1}$ Centre for Liver Research, University of Birmingham; ${ }^{2}$ Department of Surgery, Loyola University, Chicago; ${ }^{3}$ Department of Gastroenterology, Essen University, Germany; ${ }^{4}$ Division of Gastroenterology, Duke University, USA

Introduction Steatohepatitis is the critical step in the progression to fibrosis, and is characterised by increased inflammatory cell recruitment from the circulation. The cytokine Osteopontin (OPN) is intricately involved in cell-recruitment and tissue-repair, and we reported that OPN is significantly upregulated during non-alcoholic steatohepatitis (NASH).

Aim Thus, we hypothesised that OPN promotes steatohepatitis by supporting leucocyte migration across hepatic sinusoidal endothelium.

Method Wild-type mice were fed chow or methionine-choline deficient (MCD) diet to induce NASH. After 4 weeks, mice were sacrificed; severity of disease assessed by serum aminotransferase (AST), hepatic OPN quantified by ORTPCR and immunohistochemistry, serum OPN measured by ELISA. In separate experiments, MCD-fed mice were treated with anti-OPN or IgG, and flow cytometry used to quantify numbers of liver infiltrating lymphocytes (LIL). Primary human hepatic sinusoidal endothelial cells (HSEC) were stimulated with recombinant (r) OPN (0-1000 ng/ $\mathrm{ml}$ ), and expression of adhesion molecules (ICAM-1, VCAM-1, CD31) quantified by western blot. To assess lymphocyte transendothelial migration, lymphocytes were perfused over rOPN- or vehicle-treated-HSEC, with or without TNFa $(20 \mathrm{ng} / \mathrm{ml})+$ IFNa $(100 \mathrm{ng} / \mathrm{ml})$. In separate experiments, TNFa+IFNa stimulatedHSEC were treated with sham or OPN-aptamers and total lymphocyte adhesion recorded. Human livers with NASH were immunostained for OPN, and FACS used to quantify LIL isolated from control or NASH-cirrhotic patients.

Results In mice, diet-induced NASH upregulated expression of hepatic OPN by threefold $(\mathrm{p}<0.05)$, serum OPN by twofold $(p<0.05)$, and increased intrahepatic CD4 by 2.2 -fold, CD8 by $4.5-$ fold, and NKT cells by 3.2 -fold $(p<0.05)$. MCD-fed mice treated with anti-OPN accumulated fewer CD3, CD4, CD8 and NKT cells $(p<0.05)$, and exhibited attenuated injury (ALT: threefold reduction; $\mathrm{p}<0.02)$. rOPN induced expression of ICAM-1, VCAM-1 and CD31 on human HSEC, enhanced lymphocyte recruitment under conditions of flow (41\%), and amplified recruitment capacity of TNF $\alpha$ + IFN $\gamma$ stimulated HSEC (23\%), while OPN neutralisation with RNA-aptamers reduced lymphocyte recruitment by $50 \%$ (all $\mathrm{p}<0.05$ ). In humans, expression of OPN was significantly upregulated in NASH; livers from NASH-cirrhosis harboured twofold more CD4 and threefold more CD8 and NKT cells $(p<0.05)$ than normal. Conclusion OPN is upregulated during steatohepatitis in mice and humans, and promotes lymphocyte recruitment across HSEC. Neutralisation of OPN significantly reduces lymphocyte recruitment and liver injury. Our results suggest that OPN is a promising anti-inflammatory target in steatohepatitis.

\section{P90 $\propto-1$ ANTITRYPSIN (A1AT) POLYMERS CAUSE EXTREME HEPATOCYTE AGEING}

doi:10.1136/gutjnl-2011-300857a.90

${ }^{1} \mathrm{M}$ Mela, ${ }^{1} \mathrm{~S}$ Davies, ${ }^{1} \mathrm{~S}$ Verma, ${ }^{1} \mathrm{P}$ Tachtatzis, ${ }^{2} \mathrm{E}$ Miranda, ${ }^{2} \mathrm{D}$ A Lomas, ${ }^{3} \mathrm{~N}$ Coleman ${ }^{1} \mathrm{G}$ Alexander. ${ }^{1}$ Addenbrooke's Hospital; ${ }^{2}$ Clinical Institute for Medical Research, University of Cambridge; ${ }^{3}$ Department of Pathology, University of Cambridge

Introduction a1AT, synthesised predominantly in the liver, is the archetypal inhibitor of the serpin protein family. a1AT deficiency is a common inherited disorder; Glu342Lys substitution causes abnormal folding of mutant protein, which may polymerise and aggregate in the endoplasmic reticulum. a1AT aggregates are the histological hallmark of a1AT-related liver disease but it is unclear how aggregates induce liver injury.

Aim To determine whether hepatocytes containing polymeriseda1AT (pa1AT) had accelerated ageing manifest as shortened telomeres.

Method Liver biopsy sections were studied from 60 patients with a1AT- related liver disease with a broad spectrum of fibrosis, recruited from the Cambridge metabolic liver clinic (30 were homozygous and 30 heterozygous). Comparison was made with sections from 20 age and sex matched time zero biopsies obtained at liver transplant. Mean hepatocyte telomere length, a reflection of age, was measured by quantitative fluorescent in situ hybridisation (QFISH) with a PNA Cy5 probe. Nuclei were identified with DAPI, hepatocytes with antibody against hepar-1 and pa1AT with specific mouse monoclonal antibody (2C1). Images were obtained and analysed using the Olympus ScanR software system (Abstract P90 figure 1). Statistical analysis used Graph Pad Prism.

Results Hepatocyte nuclei were larger in patients with both homozygous and heterozygous a1AT deficiency $(p=0.002)$ and had shorter telomeres $(p<0.0001)$ than age and sex matched controls. Homozygous patients had shorter hepatocyte telomeres than heterozygous patients $(p=0.003)$. Hepatocyte nuclei in both homozygous and heterozygous a1AT deficiency were larger in cells with pa1AT compared to neighbouring cells without pa1AT $(p=0.002)$. Hepatocyte telomeres were far shorter in cells that contained pa1AT than neighbouring hepatocytes without pa1AT $(\mathrm{p}<0.0001$, Abstract P90 figure 2). Hepatocytes with pa1AT showed additional telomere shortening with increased age $(p=0.0002)$. Fibrosis stage was related to telomere shortening- telomeres shortened as the stage of fibrosis increased.

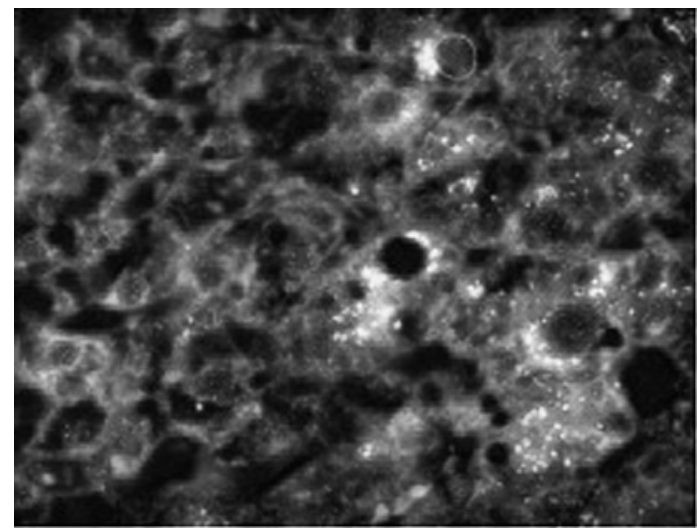

Abstract P90 Figure 1 OFISH image which highlights the a1AT polymers (shown as bright white speckles), surrounding some of the affected hepatocytes. 